\title{
Monitoring methylation-driven genes as prognostic biomarkers in patients with lung squamous cell cancer
}

\author{
PENGKAI HAN*, QIPING LIU* and JIANHUA XIANG \\ Department of Respiratory Medicine, Chongqing Three Gorges Central Hospital, Chongqing 404100, P.R. China
}

Received April 15, 2019; Accepted October 16, 2019

DOI: $10.3892 / \mathrm{ol} .2019 .11163$

\begin{abstract}
Aberrant DNA methylations have been reported to be significantly associated with lung squamous cell cancer (LUSC). The aim of this study was to investigate the DNA methylation-driven genes in LUSC by integrative bioinformatics analysis. In the present study, methylation-driven genes in LUSC were screened out, and survival analysis related to these genes was performed to confirm their value in prognostic assessment. Gene expression and methylation data were downloaded from The Cancer Genome Atlas (TCGA), and the MethylMix algorithm was used to identify methylation-driven genes. ConsensusPathDB was used to perform Gene Ontology and pathway enrichment analysis of methylation-driven genes. Survival analysis was performed to investigate the correlation with prognosis. In total, 52 differentially expressed methylation-driven genes were identified in LUSC and adjacent tissues. Survival analysis showed that $D Q X 1, G P R 75, S T X 12$, and TRIM61 could serve as independent prognostic biomarkers. In addition, the combined methylation and gene expression survival analysis revealed that the combined expression level of the genes $A L G 1 L, D Q X 1$, and ZNF418 alone can be used as a prognostic marker or drug target. Methylation of four sites of gene ZNF418, four sites of ZNF701, two sites of DQX1, and four sites of DCAF4L2 was significantly associated with survival. The present study provides an important bioinformatic and relevant theoretical basis for subsequent early diagnosis and prognostic assessment of LUSC.
\end{abstract}

\section{Introduction}

Lung cancer is a common malignant tumor, and is the leading cause of cancer-related death in the United States and

Correspondence to: Dr Jianhua Xiang, Department of Respiratory Medicine, Chongqing Three Gorges Central Hospital, 165 Xincheng Road, Wanzhou, Chongqing 404100, P.R. China

E-mail: xjh661666@163.com

${ }^{*}$ Contributed equally

Key words: lung squamous cell carcinoma, DNA methylation-driven genes, biomarkers, survival analysis worldwide (1). The cancer occurs below the bronchi, and there are no typical clinical symptoms in the early stage. According to the characteristics of pathological tissues, lung cancer can be classified into two major categories: Small cell lung cancer (SCLC) and non-small cell lung cancer (NSCLC). NSCLC is the most common subtype, accounting for $>80 \%$ of all lung cancer cases (2). The lung squamous cell cancer (LUSC) subtype accounts for $25-30 \%$ of all lung cancers, and is mainly presented as central lung cancer (3). Studies have shown that squamous cell carcinoma is associated with smoking, and is common in men $(1,2)$. However, not all smokers develop lung cancer. Only $10-20 \%$ of smokers develop lung cancer in their lifetime, which may be due to genetic differences in susceptibility (4). In recent years, great progress has been made in molecular targeted therapy for LUSC; however, further research is required. Unfortunately, some patients do not benefit from conventional chemotherapy and radiotherapy due to drug resistance and toxic effects. Studies have shown that changes in neoplasms at the molecular level occur earlier than various clinical features $(5,6)$. Therefore, research focusing on the molecular level may be more conducive to the early diagnosis and treatment of cancer to improve the prognosis. New diagnostic markers and therapeutic targets are urgently needed to further improve the diagnosis and treatment of LUSC, and to reduce the fatality rate.

Although progress continues to be made in the treatment of non-squamous NSCLC, the needs of patients with squamous NSCLC remain unmet. With regard to targeted chemotherapy drugs, there has been a focus on non-squamous cell disease in terms of most regulatory approvals for advanced NSCLC, and updates to clinical practice guidelines. Although the progress that has been made with the therapeutic strategies of squamous NSCLC is limited, the identification of more effective treatments for this patient group is gaining momentum. Therefore, further research on LUSC will provide new directions for treatment.

With the use of the current advanced DNA methylation and RNA sequence research methods, great progress has also been made in research on the relationship between DNA methylation and gene expression during the onset and infiltration of neoplasms. In a study of the integrative analysis of DNA methylation and mRNA expression, Shi et al (7) revealed the function of epigenetic changes on LUSC. However, the mechanism contributing to oncogenesis is unclear, and in view of this, Gevaert (8) developed MethylMix, a new computational algorithm implemented in $\mathrm{R}$ software, to identify abnormal methylated genes and predict changes in transcription. The 


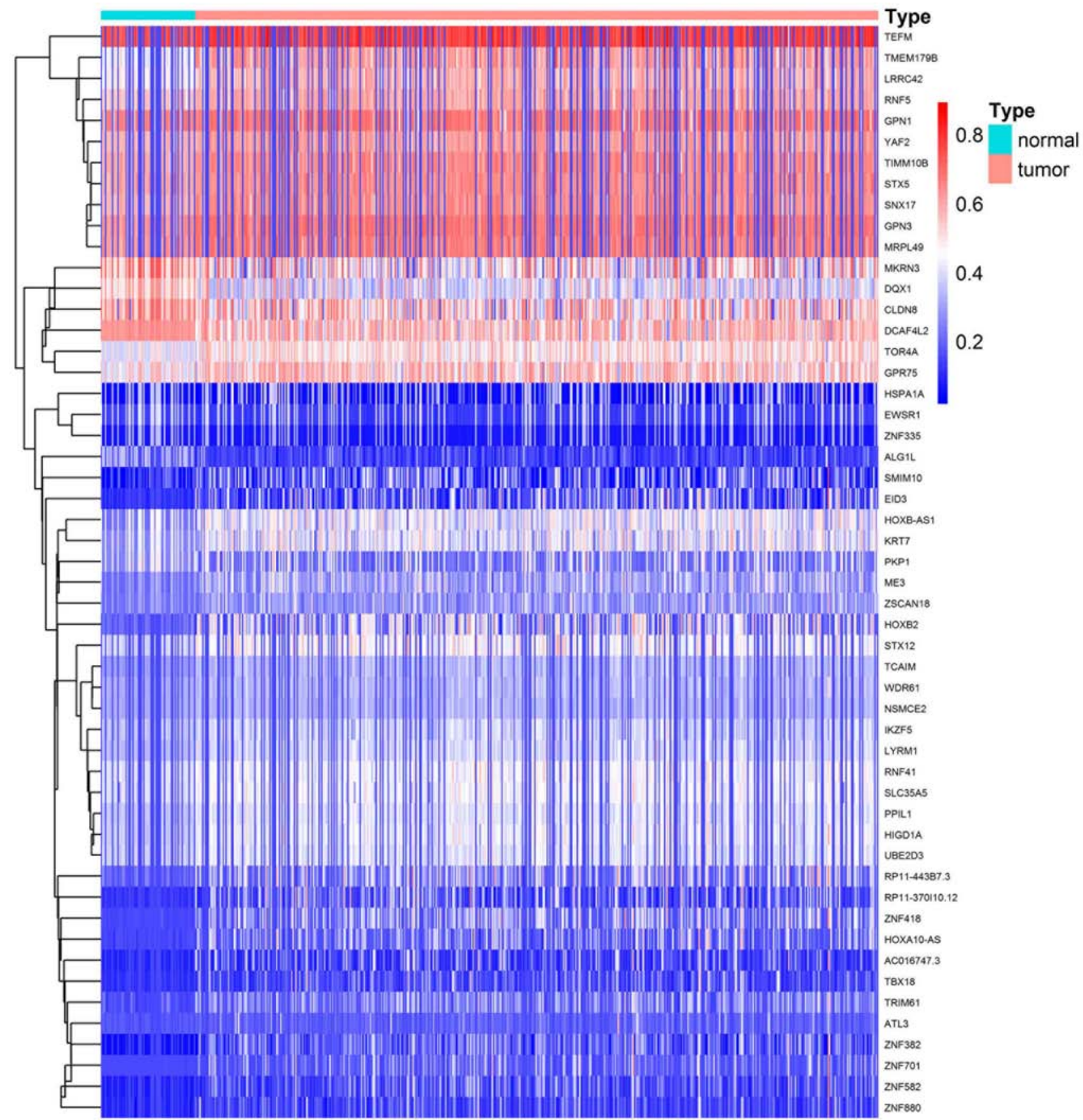

Figure 1. Heatmap of methylation-driven genes. The red color represents hypermethylation, whereas the blue color represents hypomethylation.

Cancer Genome Atlas (TCGA) (9), a well-known database on the cancer genome, provides a large amount of genetic information and clinical data, which can assist in understanding the clinical characteristics of molecular information.

In the present study, LUSC-related genes with abnormal methylation were identified in the TCGA database, and associated differential genes of abnormal methylation in LUSC were determined. Gene expression and abnormal methylation gene data of LUSC samples from the TCGA database were analyzed. Four candidate genes [DQX1 (DEAQ-box RNA dependent ATPase 1), GPR75 (probable G-protein coupled receptor 75), STX12 (syntaxin 12), and TRIM61 (putative tripartite motif-containing protein 61$)$ ] were identified from 52 methylation-driven genes $(\mathrm{P}<0.05)$, which may be independent prognostic biomarkers. Furthermore, the genes ALGIL (ALG1 chitobiosyldiphosphodolichol beta-mannosyltransferase like), $D Q X 1$, and ZNF418 (zinc finger protein 418) were confirmed to meaningfully predict prognosis by integrative survival analysis. In addition, a significant association between site methylation and survival was found.

\section{Materials and methods}

Data acquisition and preprocessing. Methylation and mRNA expression data of LUSC patients were downloaded from the TCGA database (https://portal.gdc.cancer.gov/) (accessed March, 2019) (10). The methylation data were obtained from 573 samples, including 69 normal samples and 504 cancer 
A

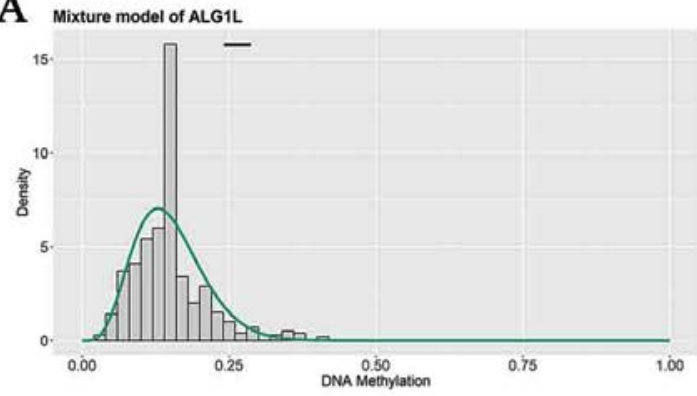

C Mixture model of ZNF701

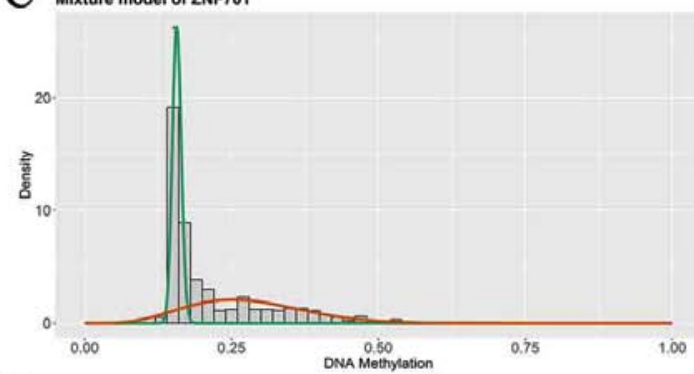

E Mixture model of DCAFAL2

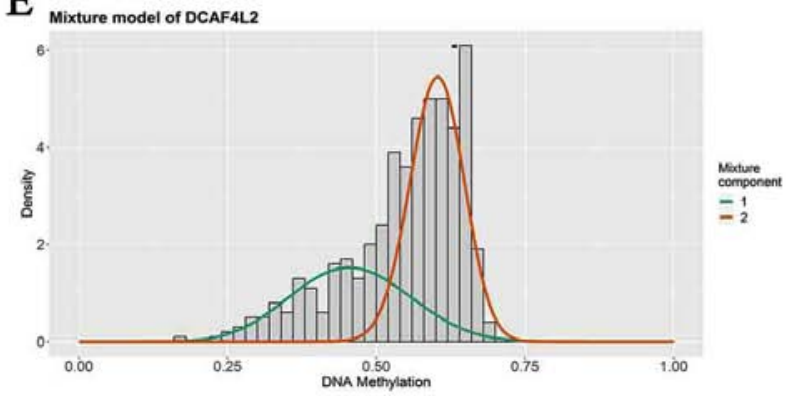

B
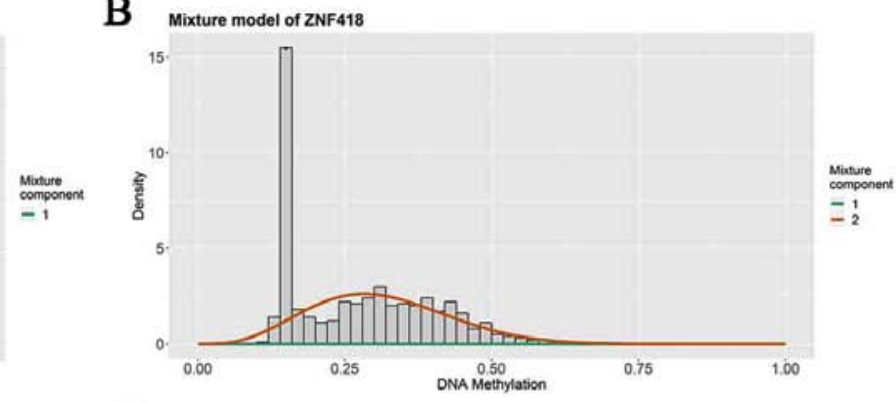

$\mathrm{D}$
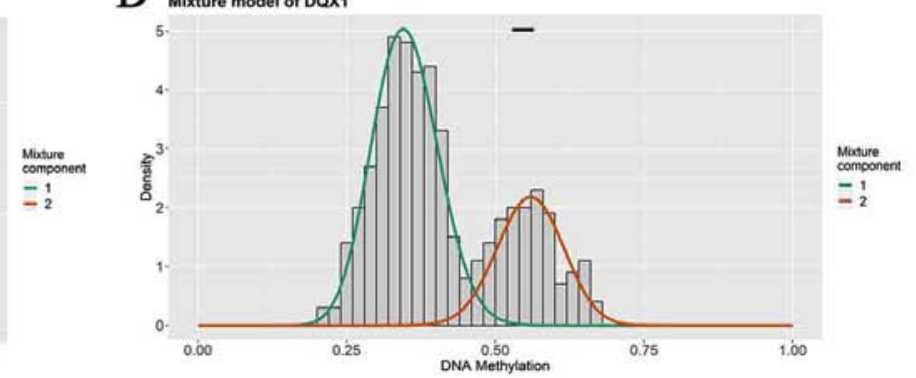

Figure 2. Distribution map of methylation states of selective genes. Mixture models of ALG1L (A), ZNF418 (B), ZNF701 (C), DQX1 (D) and DCAF4L2 (E) are presented. The horizontal axis represents the methylation level, the vertical axis represents the number of samples, the histogram represents the methylation states in the tumor group, and the curve represents the methylation distribution trend of the tumor group. The black line at the top represents the methylation state in the non-tumor group.

samples, and the mRNA expression data were obtained from 551 samples, including 49 normal samples and 502 LUSC samples. Clinical survival data were also included. The LIMMA 3.40.6 package (http://www.bioconductor.org/packages/release/bioc/html/limma.html) (11) in R language was employed to identify aberrant methylated genes and differentially expressed genes between lung cancer and normal tissues.

Identification of methylation-driven gene. The MethylMix (http://www.bioconductor.org/packages/ release/bioc/html/MethyIMix.html), an algorithm implemented by R 3.5.2 software (http://www.rproject.org/) was used to analyze the correlation between gene methylation and gene expression and to screen methylation-driven genes. The following three data files are required: DNA methylation data for normal group; DNA methylation data from cancer group; and matched gene expression data for cancer group. Based on the MethylMix algorithm, the correlation between the level of methylation and gene expression was calculated. Next, genes significantly associated were identified and the hypomethylation and hypermethylation genes were also determined by the $\beta$-mixed model. Finally, methylation-driven genes were identified $(8,12)$. $\mathrm{P}<0.05$ and $\mathrm{Cor}<0.3$ were the selected criteria for screening.
Gene Ontology $(G O)$ enrichment analysis. ConsensusPathDB (http://cpdb.molgen.mpg.de/CPDB, version 34) is an online software integrating interaction networks in Homo sapiens, including binary and complex signaling, gene regulatory and drug-target interactions, as well as biochemical pathways $(13,14)$. The methylation-driven genes list was submitted following the instructions on the website, the submit list was clicked and the data were downloaded. Finally, the GOplot R package was used to plot the enrichment results. $\mathrm{P}<0.05$ was set as the cut-off criterion for GO enrichment analysis.

Pathway analysis. ConsensusPathDB was also used to perform pathway enrichment analysis of methylation-driven genes $(14,15)$. The pathway enrichment analysis of methylation-driven genes was performed using the Kyoto Encyclopedia of Genes and Genomes (KEGG) database (http://www. genome.ad.jp/kegg/). The pathways were selected with $\mathrm{P}<0.05$ as the cut-off criterion.

Survival analysis of driver genes and methylated sites. The methylation levels of driver genes were extracted, followed by Kaplan-Meier analysis using the survival analysis package in $\mathrm{R}$ software to compare the effects of different methylation levels of driver genes on survival (16). The P-value was 

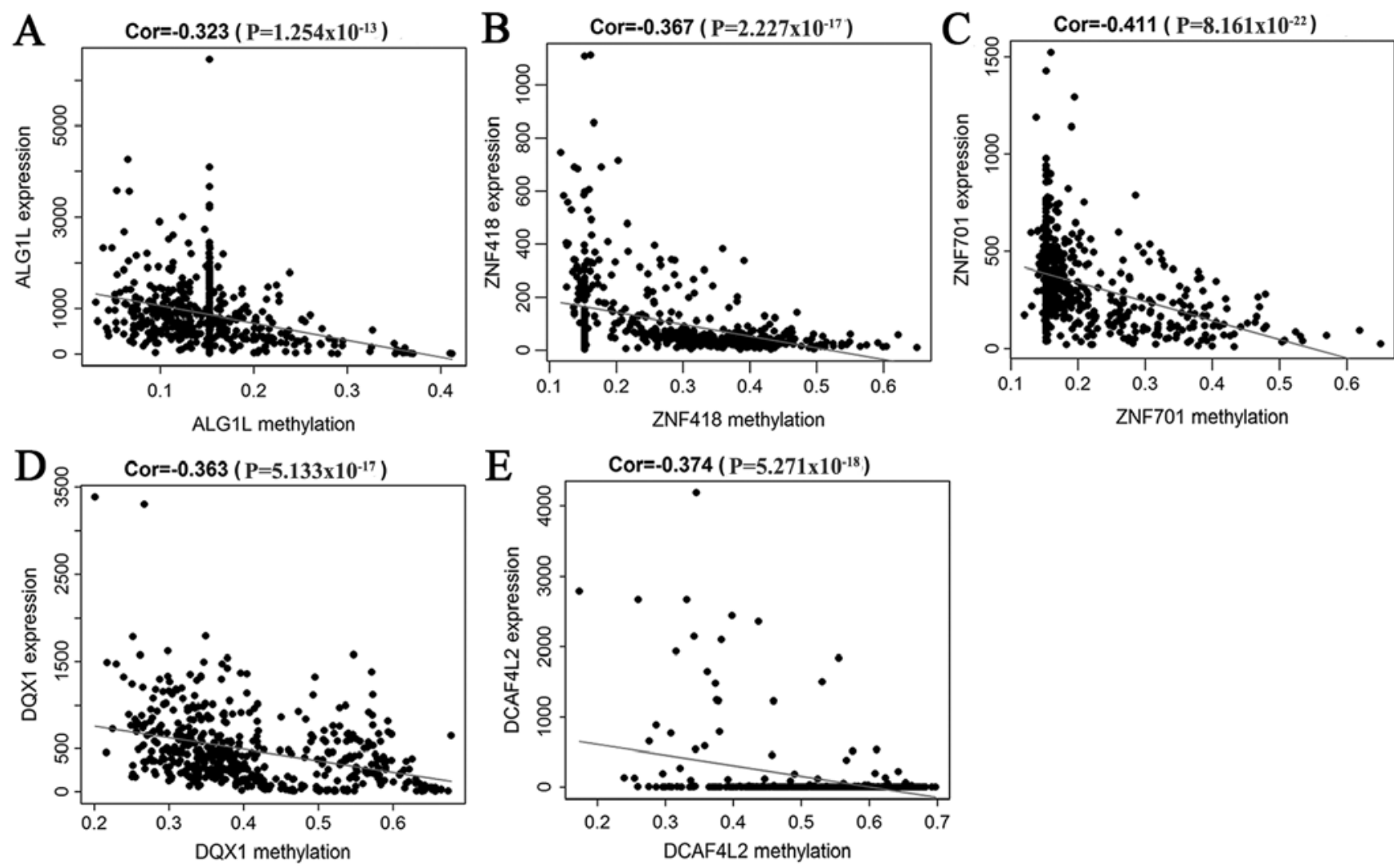

Figure 3. Correlation between the expression level of selective genes and the degree of methylation. (A-E) Pearson's correlation analysis revealed a negative correlation between gene expression and methylation for (A) ALF1L, (B) ZNF418, (C) ZNF701, (D) DQX1 and (E) DCAF4L2. The abscissa represents the methylation level, and the ordinate represents gene expression. Cor represents the correlation coefficient of Pearson's analysis.

obtained using the long-rank test. The level of gene methylation was then combined with gene expression data to analyze the combined effect on survival, which was also performed using the survival $\mathrm{R}$ package. In addition, the methylation information on the related sites of the methylation-driven genes was extracted, based on the downloaded methylation and clinical data of squamous cell carcinoma from the TCGA to further determine the value of the methylation-driven genes for prognostic evaluation. The survival curve was drawn using the survival $\mathrm{R}$ package. $\mathrm{P}<0.05$ was considered to indicate a statistically significant value.

\section{Results}

Screening of methylation-driven genes. A total of 52 methylation-driven genes were identified by comparing the levels of methylation in tumor and normal tissues, and the genes were visualized using a heatmap (Fig. 1). The methylation level of 44 genes in the cancer group was higher compared with that in the normal group, and the methylation level of 8 genes was lower compared with that of the normal group. Five methylation-driven genes with the smallest P-values were selected to plot a distribution map of the degree of methylation (Fig. 2A-E). The distribution of the remainder of the genes is shown in Fig. S1.

Correlation analysis between gene methylation and expression. A correlation analysis between the methylation level of 52 methylation-driven genes and their expression was performed, which indicated that the level of methylation was negatively correlated with the respective expression of these genes. Five genes were selected to generate their scatterplots, and to estimate the correlation coefficient. These genes were the first 5 with the smallest $\mathrm{P}$-values in the correlation test (Fig. 3A-E).

GO enrichment analysis. GO enrichment analysis was conducted using the ConsensusPathDB online software. Methylation-driven genes were enriched in 'intracellular membrane-bounded organelle', 'Smc5-Smc6 complex', and 'SUMO ligase complex' (Fig. 4).

Pathway analysis. The pathway enrichment analysis is shown in Fig. 5, and was conducted in ConsensusPathDB online. In total, 13 pathways were enriched. These genes were significantly linked to 'BARD1 signaling events', 'Nicotine Pathway (Dopaminergic Neuron)' and 'Pharmacodynamics' (Fig. 5).

Survival analysis of methylation-driven genes in LUSC. Survival analysis was statistically significant when the P-value was $<0.05$. By analyzing the degree of gene methylation, it was found that the genes DQX1, GPR75, STX12, and TRIM61 significantly affected the survival and prognosis of lung cancer patients (Fig. 6). Taking genes methylation and expression as comprehensive factors, it was confirmed that the genes $A L G 1 L, D Q X 1$, and ZNF418 were closely related to prognosis (Fig. 7). The methylation levels of multiple sites of ZNF418, ZNF701, DQX1 and DCAF4L2 were found to be correlated with patient survival (Fig. 8). 


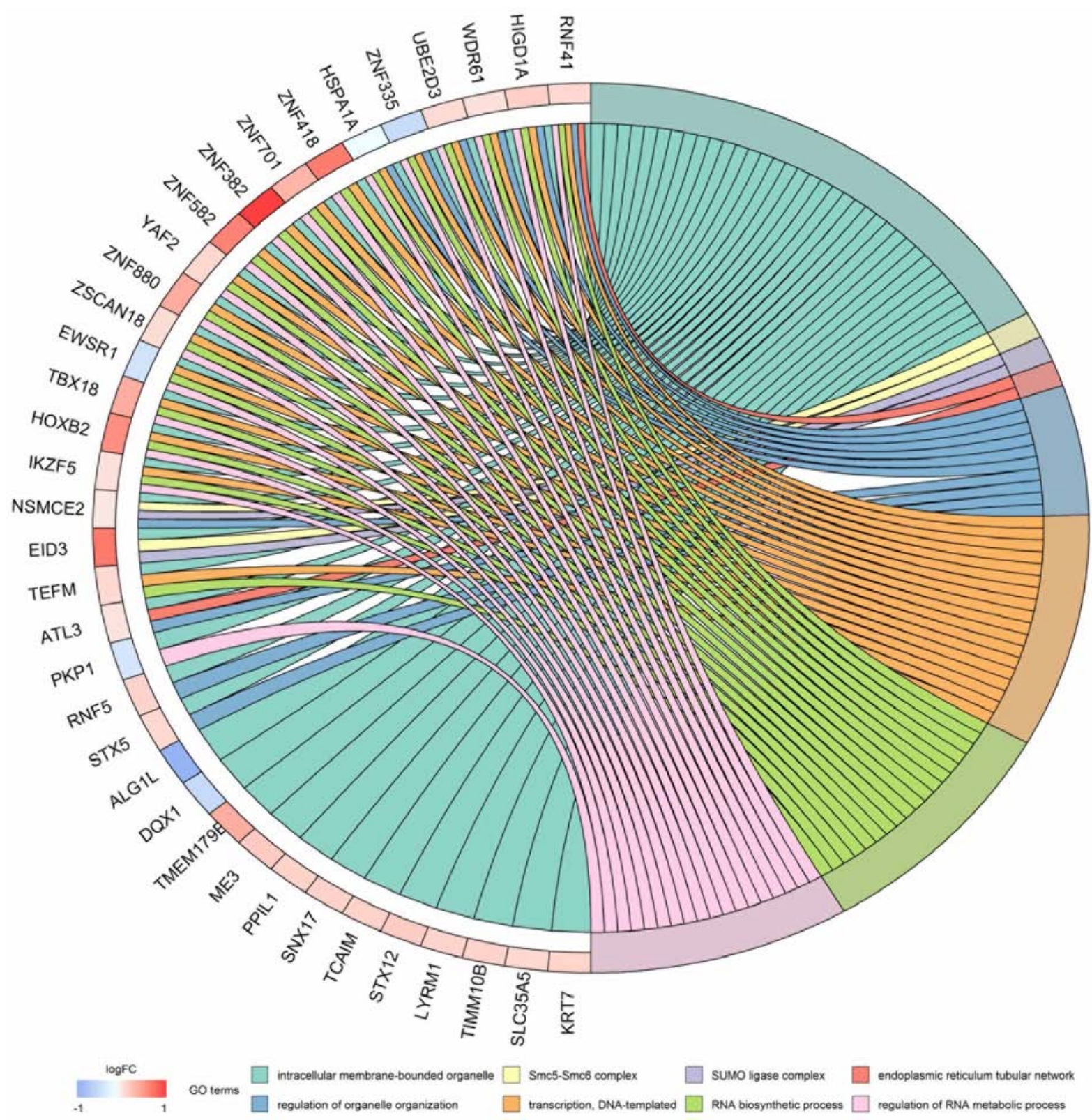

Figure 4. GO enrichment analysis of methylation-driven genes. Genes are on the left, pathways are on the right, and different pathways are presented in different colors. The color for each pathway is annotated below the ring diagram. Genes and their corresponding pathways are linked together. GO, Gene Ontology.

\section{Discussion}

LUSC, which usually has a poor prognosis, is one of the major subtypes of lung cancer, and is difficult to treat as patients tend to be older and have a higher incidence of comorbidities $(17,18)$. The occurrence of lung cancer is an intricate biological process involving multiple steps and factors, and is closely related to changes in genetic information. Epigenetic changes are involved in important aspects of lung cancer development, and its regulatory mechanisms mainly involve DNA methylation, and non-coding RNA expression regulation and histone modification (19).

In recent years, significant advances have been made in the molecular biological mechanisms of LUSC, early diagnostic evaluation markers, as well as specific genetic alterations. DNA methylation is an epigenetic mechanism that leads to tumorigenesis, and has attracted a lot of attention from researchers. DNA methylation abnormalities have been found at the genome level in the majority of tumors, including lung cancer $(20,21)$, and the frequency of $\mathrm{CpG}$ island hypermethylation in tumor cells is much higher compared with that of gene mutation (22). Abnormalities in DNA methylation often occur in the early stages of cancer, and persist throughout the development of the cancer. Methylation or demethylation may lead to tumor gene activation or inactivation of cancer suppressor genes. DNA methylation is considered to be a vitally important mechanism that causes cells to change from a normal to malignant state (23), and is the possible cause of tumor treatment tolerance (24). However, some studies have found that differentially methylated genes serve as potential cancer driver genes $(25,26)$.

Previous research findings have verified that enhanced expression of genes caused by hypomethylation, and decreased expression caused by hypermethylation, serve an important 


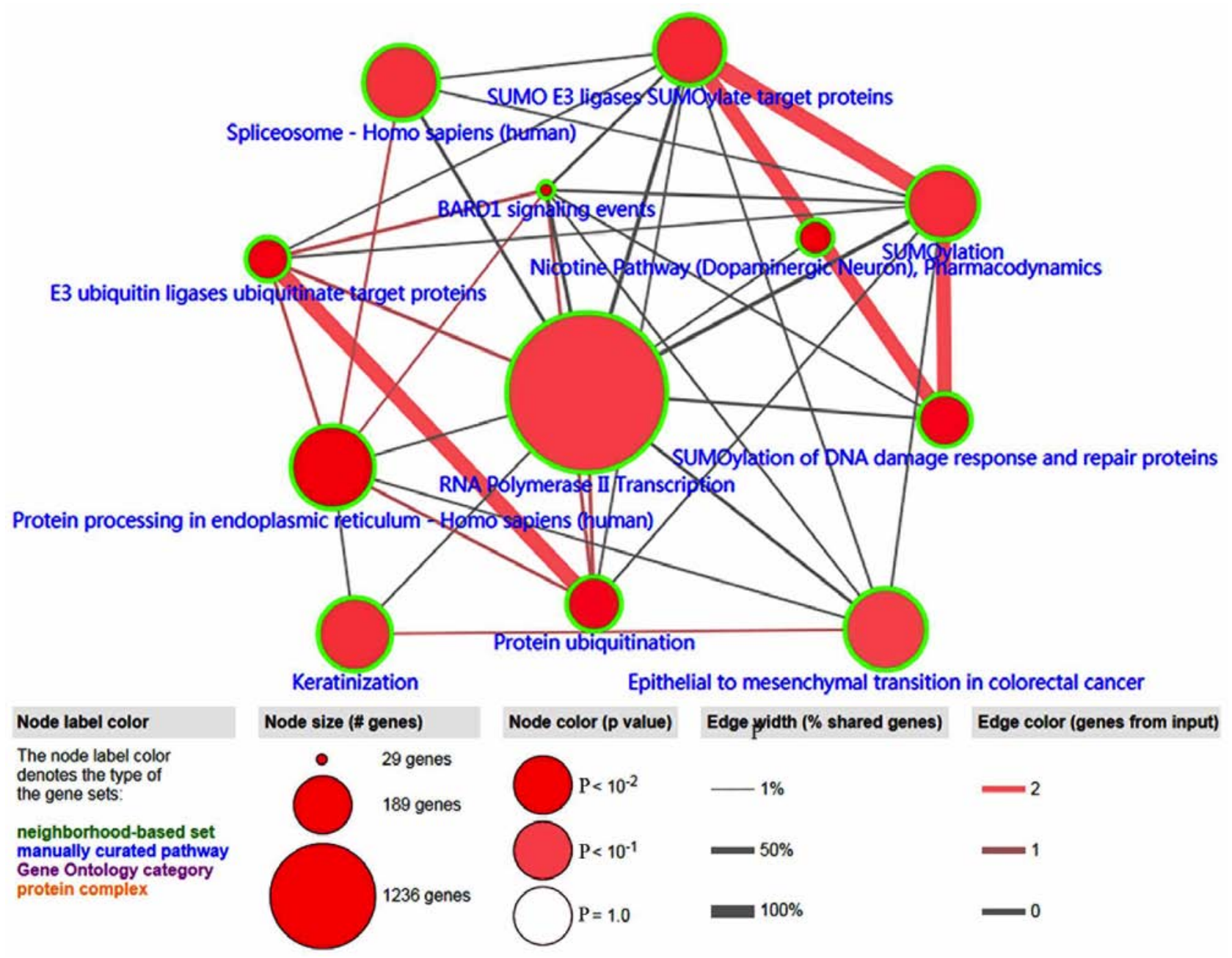

Figure 5. Pathways of significant enrichment. Every circle represents the pathway, the size of the circle represents the number of genes enriched by the pathway, and each line represents the correlation between the pathways. The color of the circle represents the P-value. Edge width represents the percentage of genes with the same pathway, and the edge color represents genes from input with the same difference before the pathway.

role in the regulation and development of malignant carcinoma (27,28). Methylation is a major epigenetic modification of genomic DNA, and an important means of regulating genomic function. Although epigenetic modifications are reversible, they have great potential as effective therapeutic targets (29). Therefore, the detection and treatment of DNA methylation as a target will result in new strategies for the diagnosis and treatment of lung cancer. For example, Sugimoto et al (30) found that patients benefit from aberrant methylation of GRWDI (glutamate rich WD repeat containing 1) in tumor development, due to activity of the GRWDl gene being inhibited by its own methylation in tumor cells, whereas expression of the GRWD1 gene can benefit tumor cell growth. In contrast, Chen et al (31) found that hypermethylation of the AGTRI promoter is more common in patients with LUSC. Ni et al (32) showed that the methylation of gene SHOX2 (short stature homeobox 1) is more pronounced in lung cancer, especially in LUSC, and is a potential non-invasive biomarker of lung cancer. A study by Guo et al (33) found that the hypermethylated state of WIF-1 gene, commonly found in NSCLC, is not only more likely to occur in squamous cell carcinoma, but its expression was also correlated with poor clinical prognosis. In a study on tumor and corresponding non-malignant lung tissue specimens, Kim et al (34) demonstrated that high methylation of the Wrap $53 \alpha$ promoter predicts a worse prognosis in patients with borderline significance. Zhang et al (35) reported that $P A X 6$ gene hypermethylation is an independent prognostic indicator, and is significantly correlated with an overall low survival rate of NSCLC, which may therefore be a potentially attractive biomarker for prognostic assessment in NSCLC patients. Thus, comprehensive functional and survival analysis of methylation-driven genes is able to provide a deeper understanding of their underlying mechanisms, and to identify novel strategies for lung cancer therapy.

In the present study, our aim was to investigate methylation-driven genes in patients with LUSC by analyzing data on gene methylation downloaded from TCGA, and to assess their relationship with prognosis. Fifty-two methylation-driven genes were identified via the MethylMix package of R software. Enrichment analysis was performed to investigate cellular functions and pathways significantly associated with these genes, and to further reveal the biological mechanism of these methylation-driven genes. Functional enrichment analysis revealed that these genes are mainly associated with 'intracellular membrane-bounded organelle', 'Smc5-Smc6 complex', and a variety of other functions, such as 'SUMO ligase 

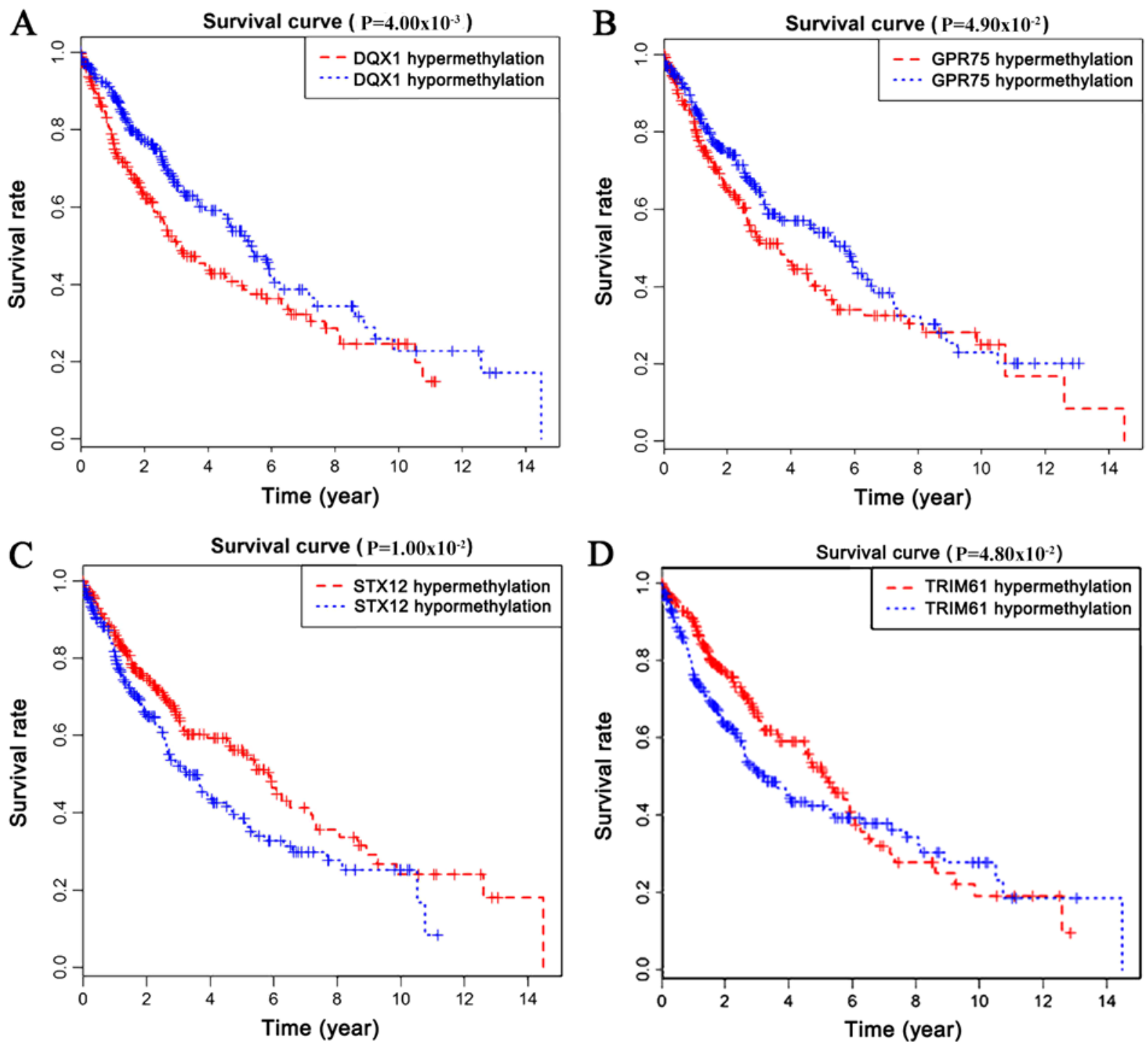

Figure 6. (A-D) Survival curves related to the methylation levels of four genes. (A) DQX1; (B) GPR75; (C) STX12; (D) TRIM6.
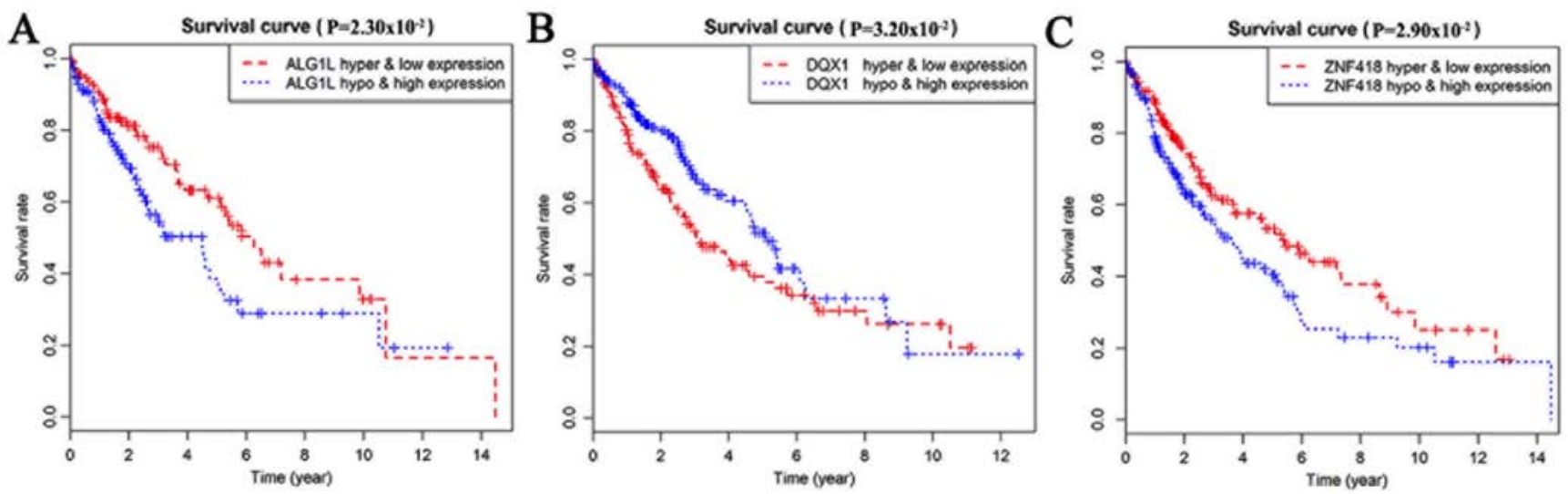

Figure 7. Survival curves related to the combination of gene expression and methylation level. (A) ALG1L; (B) DQX1; and (C) ZNF418.

complex'. These genes were found to be involved in 'BARD1 signaling events', the 'Nicotine Pathway (Dopaminergic Neuron)', and 'Pharmacodynamics' by enrichment pathway analysis. These analyses revealed the function of these genes, and their relationships with each other.
Survival analysis confirmed that methylation of the genes DXQ1, GPR75, STX12 and TRIM61 is closely related to prognosis. Survival analysis for combined gene expression and methylation indicated that $A L G 1 L, D Q X 1$, and ZNF418 could serve as markers to predict prognosis, and may even be targets 

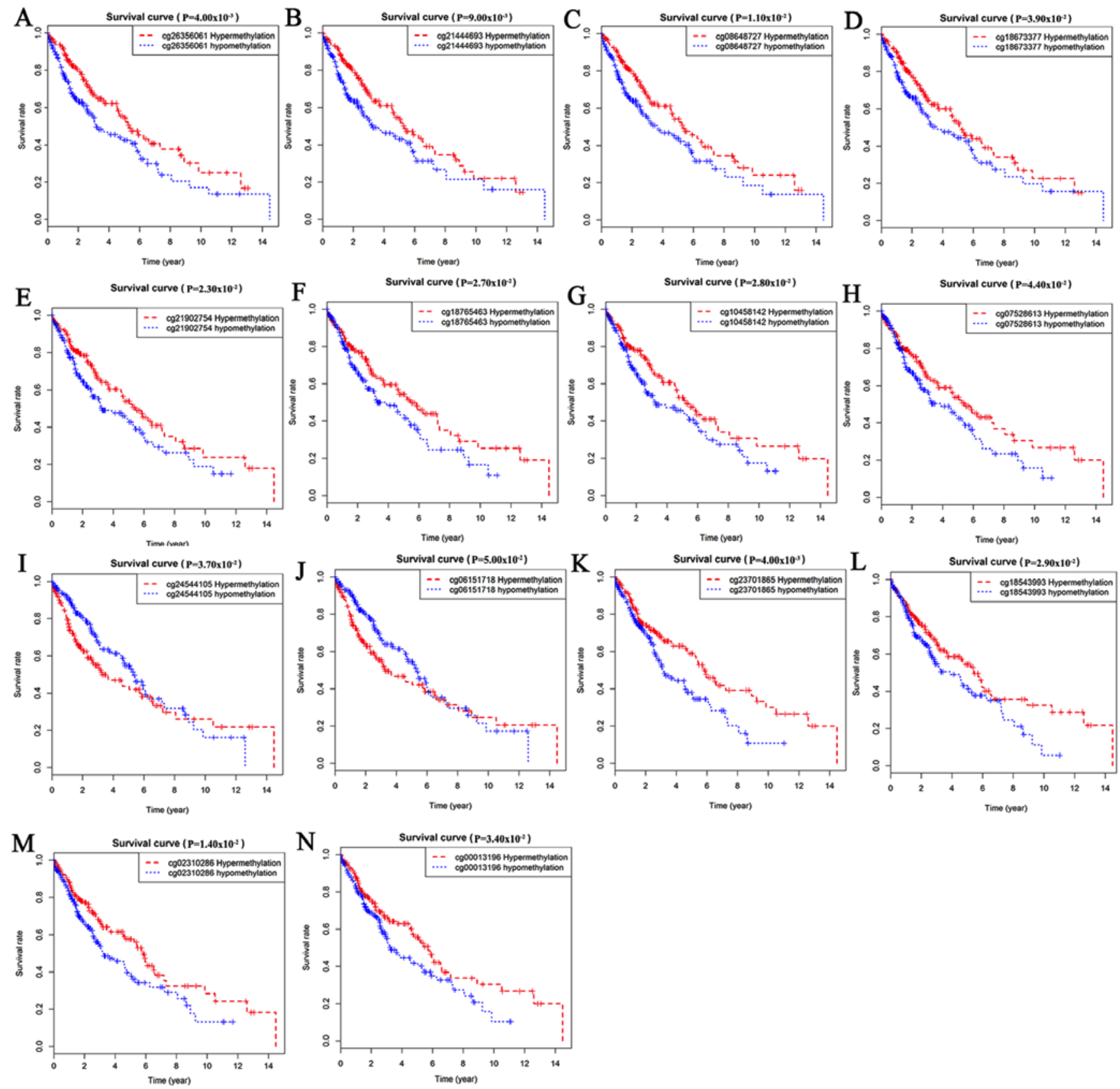

Figure 8. Survival curves related to methylation sites. (A-D) ZNF418; (E-H) ZNF701; (I and J) DQX1; and (K-N) DCAF4L2.

in future targeted therapy of LUSC. The methylation levels of multiple sites were shown to be correlated with patient survival.

A previous study found that ZNF418 is expressed in a variety of tissues, such as lung, pancreas, muscle, and heart (36). ZNF418 is significantly down-regulated in gastric carcinoma tissues (37). In the present study, integrated survival and gene expression and methylation analysis revealed that the gene ZNF418 tends to predict a poor prognosis in patients with LUSC. This suggests that ZNF418 may be involved in the mechanism of progression in LUSC. DCAF4L2 is a member of the WD-repeat domain-containing protein family, which is an intermediary of protein-protein interaction. The levels of DCAF4L2 have been previously reported to be elevated in lung cancer and in human colorectal cancer, leading to worse clinical staging, involving lymphatic and distant metastases. These findings confirm its oncogenic role (38). GPR75, a $\mathrm{G}$ protein-coupled receptor, is significantly hypermethylated in colorectal neoplasia (39). Consistently, the present study also showed that it is hypermethylated in LUSC, and this hypermethylation is associated with infaust outcome of LUSC. STX12 is a member of the syntaxin family of soluble $N$-ethylmaleimide-sensitive factor attachment protein receptors (SNAREs). A previous study reported that STX12 is able to mediate tumor cell invasion (40). The present study has revealed that the expression of $S T X 12$ is negatively correlated with methylation, and hypermethylation of STX12 is associated with favorable clinical results in LUSC. These results are theoretically consistent with LUSC; however, further validation of our findings is needed in future studies. 
The study of methylation-driven genes has important clinical significance for the early diagnosis and prognosis of lung cancer. Methylation-driven genes are expected to be identified as novel tumor markers for clinical application in the future. It is plausible that our study provides credible potential targets for the biological mechanism and clinical management of LUSC. However, further research is required to confirm our findings.

\section{Acknowledgements}

Not applicable.

\section{Funding}

The present study was funded by the Scientific Research Projects of Chongqing Municipal Health Bureau (2013-2-174), China.

\section{Availability of data and materials}

The datasets generated and/or analyzed in the current study are available from the corresponding author on reasonable request.

\section{Authors' contributions}

PH and QL drafted the manuscript, QL collected and organized the data, $\mathrm{PH}$ contributed to bioinformatics analysis by $\mathrm{R}$ software, and JX contributed to the research design. All authors read and approved the final manuscript.

\section{Ethics approval and consent to participate}

Not applicable.

\section{Patient consent for publication}

Not applicable.

\section{Competing interests}

The authors declare that they have no competing interests.

\section{References}

1. Siegel RL, Miller KD and Jemal A: Cancer statistics, 2017. CA Cancer J Clin 67: 7-30, 2017.

2. Herbst RS, Heymach JV and Lippman SM: Lung cancer. N Engl J Med 359: 1367-1380, 2008.

3. Lemjabbar-Alaoui H, Hassan OU, Yang YW and Buchanan P: Lung cancer: Biology and treatment options. Biochim Biophys Acta 1856: 189-210, 2015

4. Lorenzo-González M, Fernández-Villar A and Ruano-Ravina A: Disentangling tobacco-related lung cancer-genome-wide interaction study of smoking behavior and non-small cell lung cancer risk. J Thorac Dis 11: 10-13, 2019.

5. Jordan EJ, Kim HR, Arcila ME, Barron D, Chakravarty D Gao J, Chang MT, Ni A, Kundra R, Jonsson P, et al: Prospective comprehensive molecular characterization of lung adenocarcinomas for efficient patient matching to approved and emerging therapies. Cancer Discov 7: 596-609, 2017.

6. Aoki MN, Amarante MK, de Oliveira CEC and Watanabe MAE: Biomarkers in non-small cell lung cancer: Perspectives of individualized targeted therapy. Anticancer Agents Med Chem 18: 2070-2077, 2018.
7. Shi YX, Wang Y, Li X, Zhang W, Zhou HH, Yin JY and Liu ZQ: Genome-wide DNA methylation profiling reveals novel epigenetic signatures in squamous cell lung cancer. BMC Genomics 18 : 901, 2017.

8. Gevaert O: MethylMix: An R package for identifying DNA methylation-driven genes. Bioinformatics 31: 1839-1841, 2015.

9. Tomczak K, Czerwinska P and Wiznerowicz M: The cancer genome atlas (TCGA): An immeasurable source of knowledge. Contemp Oncol (Pozn) 19: A68-A77, 2015.

10. Grossman RL, Heath AP, Ferretti V, Varmus HE, Lowy DR, Kibbe WA and Staudt LM: Toward a shared vision for cancer genomic data. N Engl J Med 375: 1109-1112, 2016.

11. Law CW, Alhamdoosh M, Su S, Dong X, Tian L, Smyth GK and Ritchie ME: RNA-seq analysis is easy as 1-2-3 with limma, Glimma and edgeR. Version 3. F1000Res 5: 2016.

12. Cedoz PL, Prunello M, Brennan K and Gevaert O: MethylMix 2.0: An R package for identifying DNA methylation genes. Bioinformatics 34: 3044-3046, 2018.

13. Kamburov A, Pentchev K, Galicka H, Wierling C, Lehrach $\mathrm{H}$ and Herwig R: ConsensusPathDB: Toward a more complete picture of cell biology. Nucleic Acids Res 39 (Database issue): D712-D717, 2011.

14. Kamburov A, Stelzl U, Lehrach $\mathrm{H}$ and Herwig R: The ConsensusPathDB interaction database: 2013 update. Nucleic Acids Res 41 (Database issue): D793-D800, 2013.

15. Herwig R, Hardt C, Lienhard M and Kamburov A: Analyzing and interpreting genome data at the network level with ConsensusPathDB. Nat Protoc 11: 1889-1907, 2016.

16. Dudley WN, Wickham R and Coombs N: An introduction to survival statistics: Kaplan-meier analysis. J Adv Pract Oncol 7: 91-100, 2016.

17. Langer CJ, Obasaju C, Bunn P, Bonomi P, Gandara D, Hirsch FR, Kim ES, Natale RB, Novello S, Paz-Ares L, et al: Incremental innovation and progress in advanced squamous cell lung cancer: Current status and future impact of treatment. J Thorac Oncol 11: 2066-2081, 2016.

18. Paes de Araújo R, Bertoni N, Seneda AL, Felix TF, Carvalho M, Lewis KE, Hasimoto ÉN, Beckmann M, Drigo SA, Reis PP and Mur LAJ: Defining metabolic rewiring in lung squamous cell carcinoma. Metabolites 9: E47, 2019.

19. Kim D and Kim DH: Epigenome-based precision medicine in lung cancer. Methods Mol Biol 1856: 57-85, 2018.

20. Qazi TJ, Quan Z, Mir A and Qing H: Epigenetics in Alzheimer's disease: Perspective of DNA methylation. Mol Neurobiol 55: 1026-1044, 2018

21. Pan Y, Liu G, Zhou F, Su B and Li Y: DNA methylation profiles in cancer diagnosis and therapeutics. Clin Exp Med 18: 1-14, 2018.

22. Schmiemann V, Böcking A, Kazimirek M, Onofre AS, Gabbert HE, Kappes R, Gerharz CD and Grote HJ: Methylation assay for the diagnosis of lung cancer on bronchial aspirates: A cohort study. Clin Cancer Res 11: 7728-7734, 2005.

23. Baylin SB and Ohm JE: Epigenetic gene silencing in cancer a mechanism for early oncogenic pathway addiction? Nat Rev Cancer 6: 107-116, 2006.

24. Da Costa EM, McInnes G, Beaudry A and Raynal NJ: DNA methylation-targeted drugs. Cancer J 23: 270-276, 2017.

25. Manolakos A, Ochoa I, Venkat K, Goldsmith AJ and Gevaert O: CaMoDi: A new method for cancer module discovery. BMC Genomics 15 (Suppl 10): S8, 2014.

26. Gevaert O, Villalobos V, Sikic BI and Plevritis SK: Identification of ovarian cancer driver genes by using module network integration of multi-omics data. Interface Focus 3: 20130013, 2013.

27. Bernstein BE, Meissner A and Lander ES: The mammalian epigenome. Cell 128: 669-681, 2007.

28. Duruisseaux M and Esteller M: Lung cancer epigenetics: From knowledge to applications. Semin Cancer Biol 51: 116-128, 2018.

29. Jones PA, Issa JP and Baylin S: Targeting the cancer epigenome for therapy. Nat Rev Genet 17: 630-641, 2016.

30. Sugimoto N, Maehara K, Yoshida K, Yasukouchi S, Osano S, Watanabe S, Aizawa M, Yugawa T, Kiyono T, Kurumizaka H, et al: Cdt1-binding protein GRWD1 is a novel histone-binding protein that facilitates MCM loading through its influence on chromatin architecture. Nucleic Acids Res 43: 5898-5911, 2015.

31. Chen R, Hong Q, Jiang J, Chen X, Jiang Z, Wang J, Liu S, Duan S and Shi S: AGTR1 promoter hypermethylation in lung squamous cell carcinoma but not in lung adenocarcinoma. Oncol Lett 14 : 4989-4994, 2017.

32. Ni S, Ye M and Huang T: Short stature homeobox 2 methylation as a potential noninvasive biomarker in bronchial aspirates for lung cancer diagnosis. Oncotarget 8: 61253-61263, 2017. 
33. GuoH,Zhou S, TanL, WuX, WuZ and Ran R:Clinicopathological significance of WIF1 hypermethylation in NSCLC, a meta-analysis and literature review. Oncotarget 8: 2550-2557, 2017.

34. Kim DS, Lee WK and Park JY: Promoter methylation of Wrap $53 \alpha$, an antisense transcript of $\mathrm{p} 53$, is associated with the poor prognosis of patients with non-small cell lung cancer. Oncol Lett 16: 5823-5828, 2018.

35. Zhang X, Yang X, Wang J, Liang T, Gu Y and Yang D Down-regulation of PAX6 by promoter methylation is associated with poor prognosis in non small cell lung cancer. Int J Clin Exp Pathol 8: 11452-11457, 2015.

36. Dang DT, Pevsner J and Yang VW: The biology of the mammalian Krüppel-like family of transcription factors. Int J Biochem Cell Biol 32: 1103-1121, 2000.

37. Hui HX, Hu ZW, Jiang C, Wu J, Gao Y and Wang XW: ZNF418 overexpression protects against gastric carcinoma and prompts a good prognosis. Onco Targets Ther 11: 2763-2770, 2018.

38. Wang H, Chen Y, Han J, Meng Q, Xi Q, Wu G and Zhang B: DCAF4L2 promotes colorectal cancer invasion and metastasis via mediating degradation of NFkb negative regulator PPM1B Am J Transl Res 8: 405-418, 2016.
39. Ashktorab H, Daremipouran M, Goel A, Varma S, Leavitt R, Sun $\mathrm{X}$ and Brim H: DNA methylome profiling identifies novel methylated genes in African American patients with colorectal neoplasia. Epigenetics 9: 503-512, 2014

40. Williams KC and Coppolino MG: SNARE-dependent interaction of Src, EGFR and $\beta 1$ integrin regulates invadopodia formation and tumor cell invasion. J Cell Sci 127: 1712-1725, 2014.

This work is licensed under a Creative Commons Attribution-NonCommercial-NoDerivatives 4.0 International (CC BY-NC-ND 4.0) License. 